\title{
Communication \\ Association of Melanoma-Risk Variants with Primary Melanoma Tumor Prognostic Characteristics and Melanoma-Specific Survival in the GEM Study
}

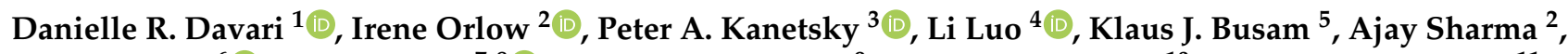 \\ Anne Kricker ${ }^{6}{ }^{(D}$, Anne E. Cust ${ }^{7,8}{ }^{\infty}$, Hoda Anton-Culver ${ }^{9}$, Stephen B. Gruber ${ }^{10}$, Richard P. Gallagher ${ }^{11}$,

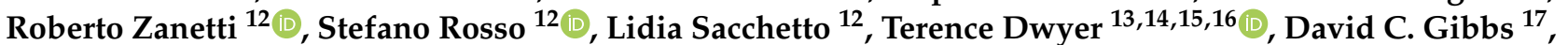 \\ David W. Ollila ${ }^{18,19}$, Colin B. Begg ${ }^{2}$, Marianne Berwick ${ }^{4}$ and Nancy E. Thomas ${ }^{1,19, *,+}$ \\ on behalf of the GEM Study Group
}

check for updates

Citation: Davari, D.R.; Orlow, I.; Kanetsky, P.A.; Luo, L.; Busam, K.J.; Sharma, A.; Kricker, A.; Cust, A.E.; Anton-Culver, H.; Gruber, S.B.; et al. Association of Melanoma-Risk Variants with Primary Melanoma Tumor Prognostic Characteristics and Melanoma-Specific Survival in the GEM Study. Curr. Oncol. 2021, 28, 4756-4771. https://doi.org/10.3390/ curroncol28060401

Received: 11 August 2021

Accepted: 10 November 2021

Published: 16 November 2021

Publisher's Note: MDPI stays neutral with regard to jurisdictional claims in published maps and institutional affiliations.

Copyright: (C) 2021 by the authors. Licensee MDPI, Basel, Switzerland. This article is an open access article distributed under the terms and conditions of the Creative Commons Attribution (CC BY) license (https:/ / creativecommons.org/licenses/by/ $4.0 /)$.
1 Department of Dermatology, University of North Carolina, Chapel Hill, NC 27599, USA; danielle_davari@med.unc.edu

2 Department of Epidemiology and Biostatistics, Memorial Sloan Kettering Cancer Center, New York, NY 10065, USA; orlowi@mskcc.org (I.O.); Ajay.sharma2@perkinelmer.com (A.S.); beggc@mskcc.org (C.B.B.)

3 Department of Cancer Epidemiology, Moffitt Cancer Center and Research Institute, Tampa, FL 33612, USA; peter.kanetsky@moffitt.org

4 Department of Internal Medicine, University of New Mexico Cancer Center, University of New Mexico, Albuquerque, NM 87102, USA; lluo@salud.unm.edu (L.L.); mberwick@salud.unm.edu (M.B.)

5 Department of Pathology, Memorial Sloan Kettering Cancer Center, New York, NY 10065, USA; busamk@mskcc.org

6 Sydney School of Public Health, The University of Sydney, Sydney, NSW 2006, Australia; anne.kricker@sydney.edu.au

7 Daffodil Centre, The University of Sydney, a Joint Venture with Cancer Council NSW, Sydney, NSW 2006, Australia; anne.cust@sydney.edu.au

8 Melanoma Institute Australia, The University of Sydney, Sydney, NSW 2065, Australia

9 Department of Medicine, University of California, Irvine, CA 92697, USA; hantoncu@uci.edu

10 City of Hope National Medical Center, Duarte, CA 91010, USA; sgruber@coh.org

11 BC Cancer and Department of Dermatology and Skin Science, University of British Columbia, Vancouver, BC V5Z 1L3, Canada; rgallagher@bccrc.ca

12 Piedmont Cancer Registry, Centre for Epidemiology and Prevention in Oncology in Piedmont, 10156 Turin, Italy; roberto.zanetti@cpo.it (R.Z.); stefano.rosso@cpo.it (S.R.); lidia.sacchetto@cpo.it (L.S.)

13 Murdoch Children's Research Institute, Melbourne, VIC 3052, Australia; terence.dwyer@wrh.ox.ac.uk

14 The Nuffield Department of Women's \& Reproductive Health, University of Oxford, Oxford OX3 9DU, UK

15 Department of Pediatrics, University of Melbourne, Melbourne, VIC 3010, Australia

16 Oxford Martin School, University of Oxford, Oxford OX1 3BD, UK

17 School of Medicine, Emory University, Atlanta, GA 30322, USA; david.corley.gibbs@emory.edu

18 Department of Surgery, University of North Carolina, Chapel Hill, NC 27599, USA; david_ollila@med.unc.edu

19 Lineberger Comprehensive Cancer Center, University of North Carolina, Chapel Hill, NC 27599, USA

* Correspondence: nancy_thomas@med.unc.edu; Tel.: +1-919-966-0785; Fax: +1-919-966-6460

+ GEM Study Group members are listed in acknowledgments.

Abstract: Genome-wide association studies (GWAS) and candidate pathway studies have identified low-penetrant genetic variants associated with cutaneous melanoma. We investigated the association of melanoma-risk variants with primary melanoma tumor prognostic characteristics and melanoma-specific survival. The Genes, Environment, and Melanoma Study enrolled 3285 European origin participants with incident invasive primary melanoma. For each of 47 melanoma-risk single nucleotide polymorphisms (SNPs), we used linear and logistic regression modeling to estimate, respectively, the per allele mean changes in log of Breslow thickness and odds ratios for presence of ulceration, mitoses, and tumor-infiltrating lymphocytes (TILs). We also used Cox proportional hazards regression modeling to estimate the per allele hazard ratios for melanoma-specific survival. Passing the false discovery threshold ( $p=0.0026)$ were associations of IRF4 rs12203592 and CCND1 rs1485993 with log of Breslow thickness, and association of TERT rs2242652 with presence of mitoses. 
IRF4 rs12203592 also had nominal associations $(p<0.05)$ with presence of mitoses and melanomaspecific survival, as well as a borderline association ( $p=0.07)$ with ulceration. CCND1 rs1485993 also had a borderline association with presence of mitoses $(p=0.06)$. MX2 rs45430 had nominal associations with log of Breslow thickness, presence of mitoses, and melanoma-specific survival. Our study indicates that further research investigating the associations of these genetic variants with underlying biologic pathways related to tumor progression is warranted.

Keywords: melanoma; single nucleotide polymorphism; Breslow thickness; ulceration; mitoses; tumor-infiltrating lymphocytes; survival

\section{Introduction}

Genome-wide association studies (GWAS) and candidate pathway studies have identified low-penetrant genetic variants associated with cutaneous melanoma [1,2]. Previously we investigated the association of 47 single nucleotide polymorphisms (SNPs) in putative melanoma-risk loci identified through GWAS or candidate studies with multiple primary melanoma occurrence and found that several of these susceptibility loci are generalizable to the risk of subsequent melanomas [3]. Many of these variants are in gene regions associated with pigmentation, such as SLC45A2, TYRP1, TYR, and ASIP [4-11]; nevi, such as NID1, MTAP, and PLA2G6 [4,6,12-18]; or both, such as IRF4 and HERC2/OCA2 [4,7,13,14,19-23]. Others are in gene regions, including $A T M$ and $M X 2$, not associated with melanoma-risk phenotypes [5]. Variants related to pigmentation and/or nevus count variation likely modify melanoma risk via these mechanisms, while others may modify risk via alternative mechanisms, such as cell proliferation $[5,24]$.

To explore whether genetic variants associated with melanoma risk could influence tumor aggressivity, we examined the associations of melanoma-risk SNPs with primary melanoma tumor prognostic characteristics. Prognostic characteristics in melanoma include Breslow thickness, ulceration, mitoses, and tumor-infiltrating lymphocytes (TILs). Breslow thickness and ulceration are the primary melanoma tumor characteristics included in the eighth edition of the American Joint Committee on Cancer staging system [25]. The presence of mitoses and a lower TIL grade are associated with worse melanoma-specific survival [26-30]. We assessed the association of melanoma-risk SNPs with log of Breslow thickness, presence of ulceration, presence of mitoses, and presence of TILs in the large, international, population-based Genes, Environment, and Melanoma (GEM) Study. To investigate whether genetic variants associated with melanoma risk could influence outcomes, we also examined the associations of these SNPs with melanoma-specific survival.

\section{Materials and Methods}

\subsection{Study Population}

The GEM Study enrolled 3579 participants with incident first- or higher-order primary cutaneous melanoma diagnosed between 1998 and 2003 in Australia, Canada, Italy, and the United States; recruitment and data collection details have been published previously [31]. Each recruitment site's institutional review board approved the study. Participants provided written informed consent. Of the 3579 patients, we limited analyses to the 3285 participants of self-reported European origin with invasive first- or higherorder primary melanoma. Twelve participants of non-European origin were excluded. An additional 282 patients with incident in situ melanoma were also excluded, as Breslow thickness, ulceration, mitoses, and TIL presence are not relevant for in situ melanomas. Thus, the final dataset for these analyses is 3285 subjects (1827 males and 1458 females) between ages 7 to 96 years old. 


\subsection{Pathology Review}

Age at diagnosis, sex, and anatomic site of the melanoma were extracted from pathology reports and confirmed during patient interview. Histologic subtype and Breslow thickness were also extracted from pathology reports. The diagnostic slides underwent centralized pathology slide review for histopathologic characteristics [30,32-34], according to established criteria $[35,36]$. The pathology slide review included evaluation of histologic subtype, Breslow thickness, ulceration, mitoses, and TIL grade. The histologic subtype from the centralized review was chosen unless missing, in which case the subtype from the pathology report was utilized. Breslow thickness was obtained from both sources, and the measure corresponding to the deepest reading was chosen to represent the value of most biological relevance. Ulceration, mitoses, and TIL grade were only obtained from the centralized review, as these characteristics are less reliably documented in pathology reports. Ulceration and mitoses were recorded as present or absent [37]. TIL grade was scored as brisk, nonbrisk, or absent using a previously defined grading system [38-40]. Missing data resulted from a lack of access to the diagnostic slide or transection of the melanoma. Breslow thickness has less missing data than ulceration, mitoses, and TIL grade because these latter characteristics were only obtained from centralized review, whereas Breslow thickness was obtained from both the centralized review and the pathology report. The pathologists conducting the centralized review were blinded to genotype and survival.

\subsection{Genotyping}

SNPs were selected, as described [3], based on their association with melanoma in other studies and genotyped from buccal swab DNA using the MassArray iPLEX assay (Agena Bioscience, San Diego, CA, USA; previously known as Sequenom) with reported quality control measures [41]. The staff running assays were blinded to outcomes.

\subsection{Survival}

Information about deaths from melanoma or other causes was obtained for all participants from National Death Indexes, cancer registries, and municipal records. Patient follow-up for vital status was complete through 2008 for British Columbia, Canada, and Turin, Italy, and to the end of 2007 for all other centers.

\subsection{Statistical Analysis}

Breslow thickness was normalized using a log transformation. Linear regression models estimated the per allele mean changes in log of Breslow thickness and 95\% confidence intervals (CIs) for each SNP. TIL grade was dichotomized as present (brisk or nonbrisk) or absent. Logistic regression models estimated the per allele odds ratios (ORs) and 95\% CIs for presence versus absence of ulceration, mitoses, or TILs for each SNP. These models were all adjusted for baseline features (age at diagnosis, sex, and study center) and lesion status as first- or higher-order primary. We performed a principal component analysis of the 47 SNPs to detect potential population structure within our data, as described previously [42].

We next explored melanoma-specific survival. For these analyses, we limited the dataset to 2458 patients of self-reported European origin who entered the study with invasive first-order primary melanoma during the ascertainment period. Patients that entered the study with second- or higher-order primary melanoma during the ascertainment period were not included. For these patients, it would have been necessary to account for previous melanomas that occurred prior to the ascertainment period, which was not included in this investigation. Survival time was accumulated from the diagnosis date until the date of death due to melanoma, date of death due to any cause other than melanoma, or the end of follow-up (censored patients). The median follow-up time was 7.7 years. Cox proportional hazards regression analyses estimated the hazard ratios (HRs) and 95\% CIs for the per allele association of each SNP with melanoma-specific survival adjusted for baseline features. In 
this analysis, for cases who developed a second primary melanoma, the occurrence of the second primary was included as a time-dependent covariate.

The false discovery threshold adjusted for multiple comparisons was computed using a resampling method that considers the linkage disequilibrium information among SNPs evaluated and is less conservative than the classical Bonferroni procedure [43,44]. All tests were two-sided. Data were analyzed using Stata/SE 16.1 (College Station, TX, USA).

\section{Results}

The demographic and tumor characteristics of the 3285 GEM participants of European origin with incident invasive primary melanoma included in these analyses are in Table 1. The median age was 58 years and $55.6 \%$ were male. Most melanomas $(43.7 \%)$ were on the trunk with smaller proportions on the head or neck (17.2\%), upper extremities $(18.1 \%)$, and lower extremities (20.9\%). The predominant subtype was superficial spreading melanoma $(65.3 \%)$. The melanomas had a median thickness of $0.70 \mathrm{~mm}$ (interquartile range $=0.44-1.26 \mathrm{~mm}) ; 6.8 \%$ had ulceration present, $32.9 \%$ had mitoses present, and $62.2 \%$ had TILs (brisk or nonbrisk TIL grade) present. The locations, minor alleles, minor allele frequencies in GEM, and literature references for the 47 SNPs are in Table S1. The numbers of samples genotyped are in Table S2.

Table 1. Characteristics of patients with incident invasive cutaneous melanoma in the GEM study $(n=3285)^{1}$.

\begin{tabular}{|c|c|}
\hline Characteristic & No. $(\%)$ \\
\hline $\begin{array}{c}\text { Median age at most recent diagnosis (IQR), } \\
\text { years }\end{array}$ & $58(46-70)$ \\
\hline \multicolumn{2}{|l|}{ Sex } \\
\hline Male & $1827(55.6)$ \\
\hline Female & $1458(44.4)$ \\
\hline \multicolumn{2}{|l|}{ Lesion status } \\
\hline First-order primary melanoma & $2458(74.8)$ \\
\hline Higher-order primary melanoma & $827(25.2)$ \\
\hline \multicolumn{2}{|l|}{ Anatomic site } \\
\hline Head/neck & $565(17.2)$ \\
\hline Trunk & $1437(43.7)$ \\
\hline Upper extremities & $595(18.1)$ \\
\hline Lower extremities & $688(20.9)$ \\
\hline \multicolumn{2}{|l|}{ Histologic subtype } \\
\hline Superficial spreading & $2144(65.3)$ \\
\hline Nodular & $275(8.4)$ \\
\hline Lentigo maligna & $377(11.5)$ \\
\hline Unclassified/other ${ }^{2}$ & 489 (14.9) \\
\hline \multicolumn{2}{|l|}{ Breslow thickness, $\mathrm{mm}$} \\
\hline Median (IQR) & $0.70(0.44-1.26)$ \\
\hline $0.01-1.00$ & $2195(66.8)$ \\
\hline $1.01-2.00$ & $592(18.0)$ \\
\hline $2.01-4.00$ & $276(8.4)$ \\
\hline$>4.00$ & $144(4.4)$ \\
\hline Missing & $78(2.4)$ \\
\hline \multicolumn{2}{|l|}{ Ulceration } \\
\hline Absent & $2392(72.8)$ \\
\hline Present & $225(6.8)$ \\
\hline Missing & $668(20.3)$ \\
\hline \multicolumn{2}{|l|}{ Mitoses } \\
\hline Absent & $1544(47.0)$ \\
\hline Present & 1081 (32.9) \\
\hline Missing & $660(20.1)$ \\
\hline
\end{tabular}


Table 1. Cont.

\begin{tabular}{cc}
\hline Characteristic & No. (\%) \\
\hline Tumor-infiltrating lymphocyte (TIL) grade & \\
Absent & $567(17.3)$ \\
Nonbrisk & $1658(50.5)$ \\
Brisk & $385(11.7)$ \\
Missing & $675(20.5)$ \\
\hline
\end{tabular}

Abbreviations: GEM, Genes, Environment and Melanoma; No., number; IQR, interquartile range. ${ }^{1}$ Limited to individuals of European origin with incident invasive first- or higher-order primary melanoma. Percentages may not sum to 100 because of rounding of decimals. ${ }^{2}$ Other includes acral lentiginous, spindle cell, nevoid, and Spitzoid melanomas.

Passing the false discovery threshold $(p=0.0026)$ were associations of IRF4 rs12203592 and CCND1 rs1485993 with log of Breslow thickness, and association of TERT rs2242652 with presence of mitoses (Table 2). Adjusting for the top two principal components from our principal component analysis did not affect these associations (OR change $0-1 \%$, results not shown). No SNPs passed false discovery for their association with presence of ulceration or TILs or melanoma-specific survival. Nominal associations $(p<0.05)$ with prognostic characteristics and melanoma-specific survival are in Tables 2 and 3, respectively.

In addition to IRF4 rs $12203592^{*} \mathrm{~T}$ passing false discovery for its association with increased log of Breslow thickness, IRF4 rs12203592*T had nominal associations $(p<0.05)$ with presence of mitoses and worse melanoma-specific survival, as well as a borderline association $(p=0.07)$ with presence of ulceration.

In addition to CCND1 rs1485993*T passing false discovery for its association with decreased log of Breslow thickness, CCND1 rs1485993* $\mathrm{T}$ was borderline associated with absence of mitoses $(p=0.06)$. Also, CCND1 rs11604821* $\mathrm{G}$ and rs11263498*T were each nominally associated with both decreased log of Breslow thickness and absence of mitoses. While TERT rs2242652 did not have any additional nominal associations, TERT rs2853676*A was nominally associated with absence of mitoses, and TERT; CLPTM1L rs401681*T was nominally associated with decreased log of Breslow thickness and absence of mitoses. MX2 rs $45430^{*} G$ had nominal associations with decreased log of Breslow thickness and absence of mitoses, as well as better melanoma-specific survival.

We have previously reported, in separate and combined analyses of GEM and the Western Australia Melanoma Health Study (WAMHS), the associations of IRF4 rs12203592, CCND1 rs11263498, and MX2 rs45430 with Breslow thickness [45] and IRF4 rs12203592 with melanoma-specific survival among first-order primary melanoma patients [46]. 
Table 2. Associations of melanoma-risk SNPs with primary melanoma tumor prognostic characteristics among patients in the GEM study ${ }^{1}$.

\begin{tabular}{|c|c|c|c|c|c|c|c|c|c|c|c|}
\hline & & & \multicolumn{9}{|c|}{ Tumor Prognostic Characteristics } \\
\hline & & & \multicolumn{2}{|c|}{ Breslow Thickness $(n=3207)$} & & \multicolumn{2}{|c|}{$\begin{array}{l}\text { Present vs. Absent } \\
\text { Ulceration }(n=2617)\end{array}$} & \multicolumn{2}{|c|}{$\begin{array}{l}\text { Present vs. Absent } \\
\text { Mitoses }(n=2625)\end{array}$} & \multicolumn{2}{|c|}{$\begin{array}{l}\text { Nonbrisk/Brisk vs. } \\
\text { Absent TIL grade } \\
\quad(n=2610)\end{array}$} \\
\hline $\begin{array}{c}\text { Gene } \\
\text { Neighborhood }\end{array}$ & SNP & $\mathrm{a} / \mathrm{A}$ & $\begin{array}{l}\text { Per allele mean change in } \\
\log \text { of Breslow thickness } \\
\qquad(95 \% \mathrm{CI})^{2}\end{array}$ & $\begin{array}{c}\text { Per allele change } \\
\text { in Breslow } \\
\text { thickness, } \%^{3}\end{array}$ & $p$ & $\begin{array}{l}\text { Per allele OR } \\
\quad(95 \% \mathrm{CI})^{4}\end{array}$ & $p$ & $\begin{array}{l}\text { Per allele OR } \\
\quad(95 \% \mathrm{CI})^{4}\end{array}$ & $p$ & $\begin{array}{l}\text { Per allele OR } \\
\quad(95 \% \mathrm{CI})^{4}\end{array}$ & $p$ \\
\hline PARP1 & rs3219090 & $\mathrm{A} / \mathrm{G}$ & $0.004(-0.04-0.05)$ & 0.44 & 0.85 & $0.96(0.77-1.19)$ & 0.70 & $0.96(0.85-1.08)$ & 0.48 & $1.07(0.93-1.25)$ & 0.34 \\
\hline PARP1 & rs2695238 & $\mathrm{C} / \mathrm{G}$ & $0.01(-0.03-0.06)$ & 1.11 & 0.62 & $0.96(0.78-1.19)$ & 0.73 & $0.97(0.86-1.09)$ & 0.60 & $1.03(0.89-1.19)$ & 0.69 \\
\hline NID1 & rs3768080 & $\mathrm{G} / \mathrm{A}$ & $-0.03(-0.07-0.006)$ & -3.35 & 0.10 & $0.83(0.68-1.01)$ & 0.06 & $0.91(0.81-1.02)$ & 0.10 & $0.95(0.83-1.08)$ & 0.42 \\
\hline NID1 & rs10754833 & $\mathrm{C} / \mathrm{T}$ & $-0.03(-0.07-0.006)$ & -3.33 & 0.10 & $0.83(0.68-1.01)$ & 0.06 & $0.90(0.81-1.01)$ & 0.08 & $0.94(0.83-1.08)$ & 0.40 \\
\hline CASP8 & $\operatorname{rs} 6735656^{a}$ & $\mathrm{G} / \mathrm{T}$ & $-0.02(-0.06-0.03)$ & -1.69 & 0.47 & $0.95(0.76-1.19)$ & 0.65 & $0.96(0.85-1.09)$ & 0.53 & $0.97(0.83-1.13)$ & 0.67 \\
\hline CASP8 & rs13016963 & $\mathrm{A} / \mathrm{G}$ & $-0.01(-0.05-0.03)$ & -1.03 & 0.62 & $1.02(0.84-1.25)$ & 0.81 & $0.91(0.81-1.02)$ & 0.10 & $1.01(0.88-1.16)$ & 0.90 \\
\hline TERT & rs2853676 & $\mathrm{A} / \mathrm{G}$ & $-0.02(-0.06-0.03)$ & -1.69 & 0.45 & $0.96(0.77-1.19)$ & 0.69 & $0.87(0.77-0.98)$ & 0.02 & $0.95(0.82-1.10)$ & 0.48 \\
\hline TERT & rs13356727 & $\mathrm{G} / \mathrm{A}$ & $-0.03(-0.07-0.007)$ & -3.29 & 0.11 & $0.92(0.76-1.12)$ & 0.41 & $0.91(0.82-1.02)$ & 0.10 & $0.92(0.81-1.06)$ & 0.24 \\
\hline TERT; CLPTM1L & rs4975616 & $\mathrm{G} / \mathrm{A}$ & $-0.03(-0.07-0.01)$ & -2.96 & 0.16 & $1.03(0.84-1.27)$ & 0.79 & $0.93(0.83-1.04)$ & 0.22 & $0.93(0.81-1.07)$ & 0.33 \\
\hline TERT; CLPTM1L & rs401681 & $\mathrm{T} / \mathrm{C}$ & $-0.05(-0.09$ to -0.007$)$ & -4.64 & 0.02 & $0.94(0.77-1.14)$ & 0.51 & $0.88(0.79-0.99)$ & 0.03 & $0.98(0.86-1.12)$ & 0.80 \\
\hline$S L C 45 A 2$ & rs16891982 & $\mathrm{C} / \mathrm{G}$ & $0.03(-0.13-0.19)$ & 2.93 & 0.72 & $1.12(0.55-2.30)$ & 0.76 & $0.73(0.47-1.14)$ & 0.16 & $0.63(0.40-0.99)$ & 0.05 \\
\hline$S L C 45 A 2$ & rs35391 & $\mathrm{T} / \mathrm{C}$ & $0.08(-0.12-0.28)$ & 8.57 & 0.41 & $1.52(0.66-3.52)$ & 0.33 & $0.91(0.53-1.57)$ & 0.73 & $0.71(0.39-1.29)$ & 0.26 \\
\hline SLC45A2 & rs26722 & $\mathrm{T} / \mathrm{C}$ & $0.04(-0.17-0.25)$ & 3.76 & 0.73 & $1.28(0.49-3.32)$ & 0.61 & $0.86(0.48-1.52)$ & 0.60 & $0.84(0.44-1.62)$ & 0.61 \\
\hline SLC45A2 & rs13289 & $\mathrm{G} / \mathrm{C}$ & $0.03(-0.01-0.07)$ & 2.87 & 0.19 & $1.11(0.90-1.35)$ & 0.33 & $1.09(0.97-1.22)$ & 0.14 & $0.82(0.72-0.94)$ & 0.005 \\
\hline IRF4 & rs12203592 & $\mathrm{T} / \mathrm{C}$ & $0.08(0.03-0.13)$ & 8.14 & 0.002 & $1.23(0.99-1.54)$ & 0.07 & $1.17(1.02-1.33)$ & 0.02 & $0.92(0.79-1.08)$ & 0.31 \\
\hline IRF4 & rs872071 & $\mathrm{A} / \mathrm{G}$ & $0.008(-0.03-0.05)$ & 0.76 & 0.71 & $0.94(0.77-1.14)$ & 0.54 & $1.05(0.94-1.17)$ & 0.42 & $1.00(0.87-1.14)$ & 0.97 \\
\hline
\end{tabular}


Table 2. Cont.

\begin{tabular}{|c|c|c|c|c|c|c|c|c|c|c|c|}
\hline \multirow[b]{3}{*}{$\begin{array}{c}\text { Gene } \\
\text { Neighborhood }\end{array}$} & \multirow[b]{3}{*}{$\mathrm{SNP}$} & & \multicolumn{9}{|c|}{ Tumor Prognostic Characteristics } \\
\hline & & & \multicolumn{2}{|c|}{ Breslow Thickness $(n=3207)$} & \multicolumn{3}{|c|}{$\begin{array}{c}\text { Present vs. Absent } \\
\text { Ulceration }(n=2617)\end{array}$} & \multicolumn{2}{|c|}{$\begin{array}{l}\text { Present vs. Absent } \\
\text { Mitoses }(n=2625)\end{array}$} & \multicolumn{2}{|c|}{$\begin{array}{l}\text { Nonbrisk/Brisk vs. } \\
\text { Absent TIL grade } \\
\quad(n=2610)\end{array}$} \\
\hline & & $\mathrm{a} / \mathrm{A}$ & $\begin{array}{l}\text { Per allele mean change in } \\
\log \text { of Breslow thickness } \\
\qquad(95 \% \mathrm{CI})^{2}\end{array}$ & $\begin{array}{c}\text { Per allele change } \\
\text { in Breslow } \\
\text { thickness, } \%{ }^{3}\end{array}$ & $p$ & $\begin{array}{l}\text { Per allele OR } \\
\quad(95 \% \mathrm{CI})^{4}\end{array}$ & $p$ & $\begin{array}{l}\text { Per allele OR } \\
\qquad(95 \% \mathrm{CI})^{4}\end{array}$ & $p$ & $\begin{array}{l}\text { Per allele OR } \\
\qquad(95 \% \mathrm{CI})^{4}\end{array}$ & $p$ \\
\hline$T Y R P 1$ & rs2733832 & $\mathrm{C} / \mathrm{T}$ & $0.02(-0.02-0.06)$ & 1.77 & 0.41 & $1.05(0.86-1.28)$ & 0.65 & $1.08(0.97-1.22)$ & 0.17 & $1.05(0.91-1.20)$ & 0.53 \\
\hline MTAP & rs2218220 & $\mathrm{T} / \mathrm{C}$ & $0.005(-0.04-0.04)$ & 0.48 & 0.82 & $0.97(0.80-1.18)$ & 0.79 & $1.01(0.90-1.12)$ & 0.92 & $1.15(1.00-1.31)$ & 0.04 \\
\hline MTAP & rs1335510 & $\mathrm{G} / \mathrm{T}$ & $0.003(-0.04-0.04)$ & 0.26 & 0.90 & $0.89(0.73-1.09)$ & 0.28 & $1.00(0.89-1.12)$ & 0.95 & $1.18(1.03-1.35)$ & 0.02 \\
\hline MTAP & rs7023329 & $\mathrm{G} / \mathrm{A}$ & $0.01(-0.03-0.05)$ & 1.22 & 0.55 & $0.98(0.80-1.19)$ & 0.82 & $0.98(0.88-1.10)$ & 0.74 & $1.10(0.96-1.26)$ & 0.16 \\
\hline MTAP & rs10811629 & $\mathrm{G} / \mathrm{A}$ & $0.006(-0.03-0.05)$ & 0.61 & 0.77 & $0.97(0.80-1.19)$ & 0.79 & $1.03(0.92-1.15)$ & 0.60 & $1.12(0.98-1.28)$ & 0.10 \\
\hline CCND1 & rs11604821 & $\mathrm{G} / \mathrm{A}$ & $-0.06(-0.11$ to -0.02$)$ & -6.06 & 0.004 & $0.98(0.79-1.21)$ & 0.84 & $0.88(0.78-0.99)$ & 0.03 & $0.98(0.85-1.12)$ & 0.73 \\
\hline CCND1 & rs11263498 & $\mathrm{T} / \mathrm{C}$ & $-0.06(-0.10$ to -0.02$)$ & -5.78 & 0.006 & $0.96(0.78-1.19)$ & 0.73 & $0.89(0.79-1.00)$ & 0.04 & $1.01(0.88-1.16)$ & 0.89 \\
\hline$T Y R$ & rs1042602 & $\mathrm{A} / \mathrm{C}$ & $0.008(-0.03-0.05)$ & 0.75 & 0.73 & $1.07(0.87-1.32)$ & 0.50 & $0.94(0.84-1.06)$ & 0.31 & $1.08(0.94-1.25)$ & 0.27 \\
\hline$T Y R$ & rs10765198 & $\mathrm{C} / \mathrm{T}$ & $0.01(-0.03-0.06)$ & 1.40 & 0.52 & $0.95(0.77-1.17)$ & 0.62 & $1.12(1.00-1.26)$ & 0.05 & $0.98(0.85-1.12)$ & 0.72 \\
\hline$T Y R$ & rs1847142 & $\mathrm{A} / \mathrm{G}$ & $0.01(-0.03-0.05)$ & 1.30 & 0.54 & $1.02(0.84-1.25)$ & 0.82 & $1.08(0.97-1.21)$ & 0.17 & $0.94(0.82-1.08)$ & 0.41 \\
\hline$T Y R$ & rs10830253 & $\mathrm{G} / \mathrm{T}$ & $0.01(-0.03-0.05)$ & 0.98 & 0.65 & $1.01(0.82-1.24)$ & 0.92 & $1.08(0.96-1.21)$ & 0.19 & $0.93(0.81-1.07)$ & 0.29 \\
\hline ATM & rs12278954 b & $\mathrm{A} / \mathrm{C}$ & $0.02(-0.04-0.07)$ & 1.59 & 0.59 & $1.04(0.79-1.37)$ & 0.76 & $0.92(0.79-1.08)$ & 0.31 & $0.99(0.82-1.20)$ & 0.94 \\
\hline$O C A 2$ & rs1800407 & $\mathrm{A} / \mathrm{G}$ & $0.004(-0.07-0.07)$ & 0.41 & 0.91 & $0.99(0.71-1.40)$ & 0.97 & $0.92(0.76-1.12)$ & 0.42 & $0.88(0.71-1.11)$ & 0.28 \\
\hline OCA2 & rs1800401 & $\mathrm{T} / \mathrm{C}$ & $-0.02(-0.12-0.07)$ & -2.37 & 0.61 & $1.06(0.68-1.67)$ & 0.80 & $1.08(0.83-1.40)$ & 0.56 & $1.23(0.89-1.70)$ & 0.22 \\
\hline HERC2 & rs1129038 & $\mathrm{G} / \mathrm{A}$ & $0.02(-0.03-0.07)$ & 2.29 & 0.37 & $1.15(0.91-1.45)$ & 0.26 & $0.97(0.85-1.12)$ & 0.72 & $0.93(0.79-1.10)$ & 0.39 \\
\hline HERC2 & rs12913832 & $\mathrm{A} / \mathrm{G}$ & $0.02(-0.03-0.07)$ & 2.03 & 0.42 & $1.12(0.89-1.42)$ & 0.34 & $0.96(0.84-1.10)$ & 0.60 & $0.95(0.80-1.11)$ & 0.51 \\
\hline ASIP & rs17305657 & $\mathrm{C} / \mathrm{T}$ & $-0.03(-0.10-0.03)$ & -3.36 & 0.31 & $0.77(0.54-1.10)$ & 0.15 & $0.87(0.72-1.05)$ & 0.14 & $1.11(0.89-1.39)$ & 0.35 \\
\hline
\end{tabular}


Table 2. Cont

\section{Tumor Prognostic Characteristics}

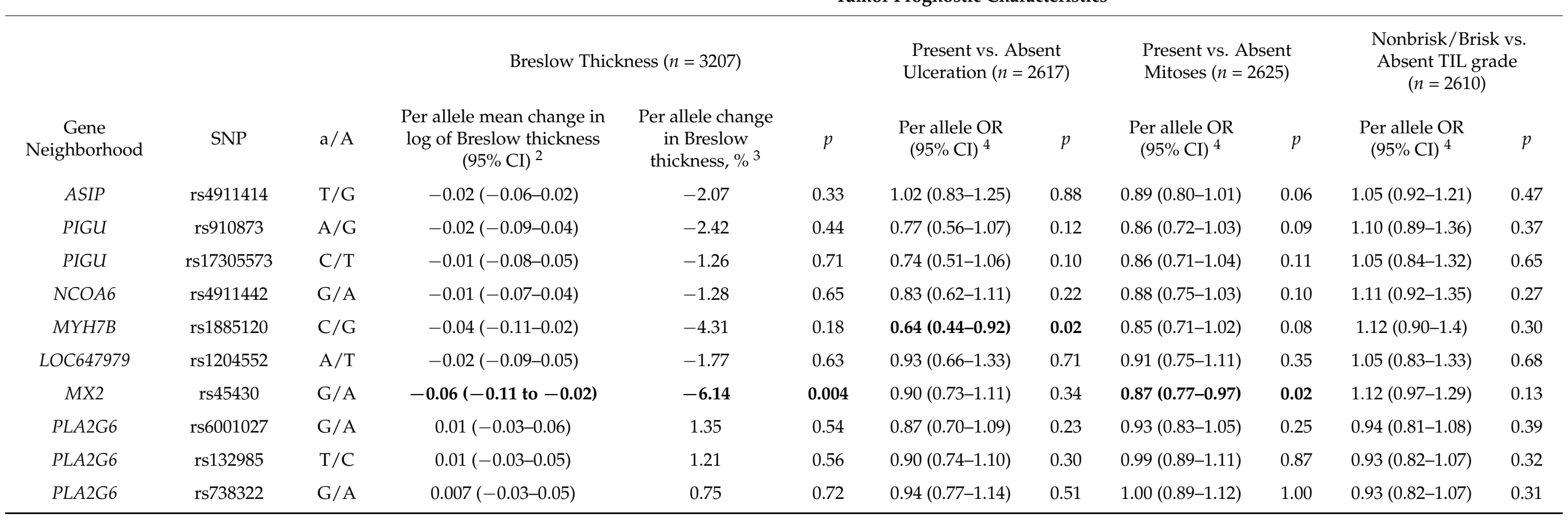

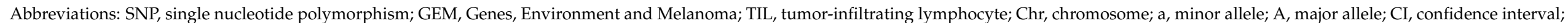

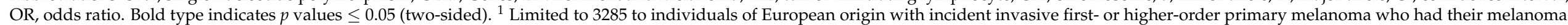

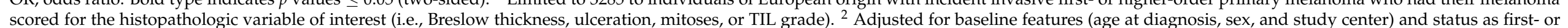

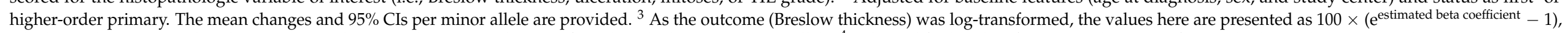

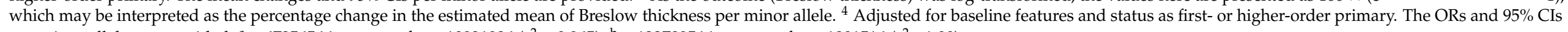
per minor allele are provided. ${ }^{a}$ rs6735656 is a proxy for $\operatorname{rs} 10931936\left(\mathrm{r}^{2}=0.965\right) .{ }^{\mathrm{b}} \mathrm{rs} 12278954$ is a proxy for $\mathrm{rs} 1801516\left(\mathrm{r}^{2}=1.00\right)$. 
Table 3. Associations of melanoma-risk SNPs with melanoma-specific survival among patients in the GEM study ${ }^{1}$.

\begin{tabular}{|c|c|c|c|c|c|c|c|}
\hline & & & Total & Censored & $\begin{array}{c}\text { Death as a Result } \\
\text { of Melanoma }\end{array}$ & \multicolumn{2}{|c|}{ Melanoma-Specific Survival } \\
\hline $\begin{array}{c}\text { Gene } \\
\text { Neighborhood }\end{array}$ & SNP & $\mathrm{a} / \mathrm{A}$ & No. & No. & No. & Per allele HR $(95 \% \mathrm{CI})^{2}$ & $p$ \\
\hline ARNT & rs7412746 & $\mathrm{C} / \mathrm{T}$ & 2420 & 2262 & 158 & $1.02(0.82-1.28)$ & 0.84 \\
\hline PARP1 & rs3219090 & $\mathrm{A} / \mathrm{G}$ & 2387 & 2232 & 155 & $1.18(0.94-1.50)$ & 0.16 \\
\hline PARP1 & rs2695238 & $\mathrm{C} / \mathrm{G}$ & 2428 & 2267 & 161 & 1.07 (0.85-1.35) & 0.58 \\
\hline NID1 & rs3768080 & $\mathrm{G} / \mathrm{A}$ & 2409 & 2251 & 158 & $0.82(0.66-1.02)$ & 0.08 \\
\hline NID1 & rs10754833 & $\mathrm{C} / \mathrm{T}$ & 2419 & 2260 & 159 & $0.83(0.66-1.03)$ & 0.09 \\
\hline CASP8 & rs6735656 ${ }^{a}$ & $\mathrm{G} / \mathrm{T}$ & 2400 & 2244 & 156 & $0.94(0.73-1.21)$ & 0.64 \\
\hline CASP8 & rs13016963 & $\mathrm{A} / \mathrm{G}$ & 2423 & 2264 & 159 & $0.93(0.75-1.17)$ & 0.55 \\
\hline TERT & rs2242652 & $\mathrm{T} / \mathrm{C}$ & 2305 & 2153 & 152 & $0.96(0.73-1.28)$ & 0.80 \\
\hline TERT & rs2853676 & $\mathrm{A} / \mathrm{G}$ & 2420 & 2259 & 161 & $0.96(0.76-1.22)$ & 0.73 \\
\hline TERT & rs13356727 & $\mathrm{G} / \mathrm{A}$ & 2439 & 2279 & 160 & $0.94(0.75-1.17)$ & 0.59 \\
\hline TERT; CLPTM1L & rs4975616 & $\mathrm{G} / \mathrm{A}$ & 2343 & 2193 & 150 & $0.94(0.75-1.19)$ & 0.61 \\
\hline TERT; CLPTM1L & rs401681 & $\mathrm{T} / \mathrm{C}$ & 2408 & 2249 & 159 & $0.97(0.77-1.21)$ & 0.76 \\
\hline SLC $45 A 2$ & rs16891982 & $\mathrm{C} / \mathrm{G}$ & 2425 & 2265 & 160 & $1.29(0.65-2.57)$ & 0.46 \\
\hline SLC45A2 & rs35391 & $\mathrm{T} / \mathrm{C}$ & 2411 & 2254 & 157 & $0.75(0.25-2.31)$ & 0.62 \\
\hline SLC45A2 & rs26722 & $\mathrm{T} / \mathrm{C}$ & 2397 & 2239 & 158 & $1.36(0.56-3.30)$ & 0.49 \\
\hline SLC45A2 & rs13289 & $\mathrm{G} / \mathrm{C}$ & 2413 & 2252 & 161 & $0.82(0.65-1.04)$ & 0.10 \\
\hline IRF4 & rs12203592 & $\mathrm{T} / \mathrm{C}$ & 2425 & 2265 & 160 & $1.28(1.00-1.65)$ & 0.05 \\
\hline IRF4 & rs872071 & $\mathrm{A} / \mathrm{G}$ & 2406 & 2247 & 159 & $0.95(0.76-1.18)$ & 0.63 \\
\hline$T Y R P 1$ & rs1408799 & $\mathrm{T} / \mathrm{C}$ & 2401 & 2242 & 159 & $1.17(0.93-1.46)$ & 0.18 \\
\hline$T Y R P 1$ & rs2733832 & $\mathrm{C} / \mathrm{T}$ & 2405 & 2248 & 157 & $1.23(0.98-1.53)$ & 0.07 \\
\hline MTAP & rs2218220 & $\mathrm{T} / \mathrm{C}$ & 2419 & 2258 & 161 & $1.05(0.84-1.30)$ & 0.68 \\
\hline MTAP & rs1335510 & $\mathrm{G} / \mathrm{T}$ & 2404 & 2249 & 155 & $0.98(0.78-1.23)$ & 0.87 \\
\hline MTAP & rs7023329 & $\mathrm{G} / \mathrm{A}$ & 2401 & 2244 & 157 & 1.05 (0.84-1.30) & 0.69 \\
\hline MTAP & rs10811629 & $\mathrm{G} / \mathrm{A}$ & 2414 & 2255 & 159 & $1.00(0.80-1.25)$ & 1.00 \\
\hline CCND1 & rs11604821 & $\mathrm{G} / \mathrm{A}$ & 2427 & 2269 & 158 & $1.02(0.81-1.29)$ & 0.86 \\
\hline CCND1 & rs1485993 & $\mathrm{T} / \mathrm{C}$ & 2410 & 2250 & 160 & $1.13(0.90-1.42)$ & 0.28 \\
\hline CCND1 & rs11263498 & $\mathrm{T} / \mathrm{C}$ & 2421 & 2263 & 158 & $1.12(0.89-1.40)$ & 0.35 \\
\hline$T Y R$ & rs1042602 & $\mathrm{A} / \mathrm{C}$ & 2429 & 2270 & 159 & $0.90(0.71-1.14)$ & 0.38 \\
\hline$T Y R$ & rs10765198 & $\mathrm{C} / \mathrm{T}$ & 2428 & 2270 & 158 & $0.90(0.71-1.14)$ & 0.37 \\
\hline$T Y R$ & rs1847142 & $\mathrm{A} / \mathrm{G}$ & 2424 & 2264 & 160 & $0.91(0.73-1.15)$ & 0.45 \\
\hline$T Y R$ & rs10830253 & $\mathrm{G} / \mathrm{T}$ & 2403 & 2247 & 156 & $0.92(0.73-1.16)$ & 0.48 \\
\hline ATM & rs12278954 b & $\mathrm{A} / \mathrm{C}$ & 2429 & 2268 & 161 & 1.37 (1.04-1.80) & 0.03 \\
\hline OCA2 & rs1800407 & $\mathrm{A} / \mathrm{G}$ & 2429 & 2270 & 159 & $1.45(1.02-2.04)$ & 0.04 \\
\hline OCA2 & rs1800401 & $\mathrm{T} / \mathrm{C}$ & 2434 & 2273 & 161 & $0.65(0.34-1.21)$ & 0.17 \\
\hline HERC2 & rs1129038 & $\mathrm{G} / \mathrm{A}$ & 2409 & 2252 & 157 & $1.38(1.07-1.77)$ & 0.01 \\
\hline HERC2 & rs12913832 & $\mathrm{A} / \mathrm{G}$ & 2429 & 2268 & 161 & $1.38(1.08-1.76)$ & 0.01 \\
\hline ASIP & rs17305657 & $\mathrm{C} / \mathrm{T}$ & 2417 & 2257 & 160 & $0.98(0.67-1.43)$ & 0.92 \\
\hline ASIP & rs4911414 & $\mathrm{T} / \mathrm{G}$ & 2426 & 2265 & 161 & $0.83(0.66-1.05)$ & 0.12 \\
\hline PIGU & rs910873 & $\mathrm{A} / \mathrm{G}$ & 2431 & 2271 & 160 & $0.99(0.70-1.41)$ & 0.96 \\
\hline PIGU & rs17305573 & $\mathrm{C} / \mathrm{T}$ & 2143 & 2003 & 140 & $1.00(0.68-1.47)$ & 0.99 \\
\hline NCOA6 & rs4911442 & $\mathrm{G} / \mathrm{A}$ & 2399 & 2241 & 158 & $1.00(0.73-1.38)$ & 0.98 \\
\hline MYH7B & rs1885120 & $\mathrm{C} / \mathrm{G}$ & 2417 & 2259 & 158 & $0.95(0.65-1.38)$ & 0.79 \\
\hline LOC647979 & rs1204552 & $\mathrm{A} / \mathrm{T}$ & 2356 & 2202 & 154 & 1.09 (0.74-1.62) & 0.67 \\
\hline MX2 & rs45430 & $\mathrm{G} / \mathrm{A}$ & 2421 & 2259 & 162 & $0.79(0.62-0.99)$ & 0.05 \\
\hline PLA2G6 & rs6001027 & $\mathrm{G} / \mathrm{A}$ & 2281 & 2133 & 148 & $1.17(0.92-1.48)$ & 0.20 \\
\hline PLA2G6 & rs132985 & $\mathrm{T} / \mathrm{C}$ & 2422 & 2263 & 159 & $1.07(0.85-1.33)$ & 0.57 \\
\hline PLA2G6 & rs738322 & G/A & 2412 & 2254 & 158 & $1.14(0.91-1.42)$ & 0.25 \\
\hline
\end{tabular}

Abbreviations: SNP, single nucleotide polymorphism; GEM, Genes, Environment and Melanoma; Chr, chromosome; a, minor allele; A, major allele; CI, confidence interval; HR; hazard ratio. Bold type indicates $p$ values $\leq 0.05$ (two-sided). ${ }^{1}$ Limited to 2458 individuals of European origin with incident invasive first-order primary melanoma. ${ }^{2}$ Adjusted for baseline features (age at diagnosis, sex, and study center) and a time-dependent covariate. The HRs and 95\% CIs per minor allele are provided. ${ }^{\text {a }}$ rs 6735656 is a proxy for rs 10931936 $\left(r^{2}=0.965\right) .{ }^{b}$ rs12278954 is a proxy for $r s 1801516\left(r^{2}=1.00\right)$.

\section{Discussion}

Our results indicate that many of these 47 melanoma-risk SNPs are not significantly associated with tumor prognostic characteristics or melanoma-specific survival when considering false discovery. Similarly, Mangantig et al., in a GWAS meta-analysis, found no significant associations with log of Breslow thickness for the ARNT, PARP1, NID1, TERT, SLC45A2, MTAP, TYR, OCA2, HERC2, ASIP, PIGU, or PLA2G6 variants we studied [47]. Mangantig et al. also found no significant association with log of Breslow thickness for 
CCND1 rs11263498 [47], while this SNP was nominally associated with log of Breslow thickness in GEM.

Consistent with GEM, Mangantig et al. found IRF4 rs12203592*T was positively associated with increased log of Breslow thickness, although not reaching genome-wide significance [47]. Similarly consistent with GEM, Potrony et al. found that IRF4 rs12203592*T increased the risk of dying from melanoma in patients from two European hospitals [48]. The IRF4 rs12203592*T allele was the melanoma-risk allele in two US studies [22,23], while it was protective in a Spanish population [20] as well as a combined analysis of Australian, UK, and Swedish subjects [21]. Here, we report the overall positive associations of IRF4 rs12203592*T with increased log of Breslow thickness, presence of mitoses, and worse melanoma-specific survival, along with a borderline association with presence of ulceration. IRF4 is a transcription factor required for the maturation of B and T cells and for the differentiation of B lymphocytes into plasma cells [49]. In immune cells, the IRF4 rs12203592*T allele increases IRF4 expression, which upregulates telomerase activity by activating TERT transcription [50-52]. Furthermore, it has been suggested that increased IRF4 expression in immune cells increases the ability of regulatory T cells to suppress $\mathrm{T}_{\mathrm{H}} 2$ responses [53], which may accelerate tumor growth. However, it has also been shown that IRF4 overexpression in myeloid-derived suppressor cells induces a decreased suppressive effect on CD8+ T cell proliferation, resulting in less rapid tumor progression [54,55].

The melanoma-risk CCND1 rs1485993*T allele [5] was positively associated with decreased Breslow thickness, passing false discovery, and borderline associated with absence of mitoses. The melanoma-risk alleles of other SNPs in the CCND1 gene neighborhood (rs11604821*G and rs11263498*T) [5] were nominally associated with both decreased Breslow thickness and absence of mitoses. These results are plausible based on CCND1's impact on cell proliferation [56]. CCND1 is a cyclin that associates with CDK4 or CDK6 to inactivate the cell cycle inhibiting the function of the retinoblastoma protein (pRB), which promotes progression through the $G_{1}-S$ phase of the cell cycle $[56,57]$. It is interesting that the melanoma-risk alleles were associated with decreased Breslow thickness and absence of mitoses. This indicates that while these variants are related to increased melanoma susceptibility, they may also be associated with decreased tumor aggressivity. A recent meta-analysis investigating the associations of CCND1 and cyclin protein D1 with melanoma prognostic factors found that upregulation of CCND1/cyclin D1 was associated with the presence of ulceration and mitoses, while the associations with Breslow thickness and survival conflicted across studies [58]. However, the associations of CCND1 rs1485993*T, CCND1 rs11604821*G, and CCND1 rs11263498*T with CCND1 expression remain unknown, and thus, we are unable to establish whether our results are consistent with the prior studies evaluating prognostic factors in the context of CCND1 expression.

The melanoma-risk TERT rs2242652*T allele [24] was positively associated with absence of mitoses, passing false discovery. The melanoma-risk TERT rs2853676*A allele [24] was also nominally associated with absence of mitoses, and the melanoma-risk rs401681*T allele $[5,24,59,60]$ was nominally associated with decreased log of Breslow thickness and absence of mitoses. These results are reasonable based on TERT's regulation of telomerase activity [61]. Again, it is notable that the melanoma-risk alleles were associated with decreased Breslow thickness and/or absence of mitoses. Activating TERT promoter mutations result in increased gene expression and have been associated with increased Breslow thickness and the presence of ulceration and mitoses in melanoma patients [62-66]. Other studies have not found associations between TERT promoter mutations and Breslow thickness, ulceration, or mitotic rate [67-69]. However, similarly to CCND1, the associations of our genotypes with TERT expression remain unknown, and thus, we are unable to establish whether our results are consistent with these prior studies.

Also noteworthy are the results that the melanoma-risk MX2 rs45430*A allele [5] was positively associated with increased Breslow thickness, presence of mitoses, and worse melanoma-specific survival in GEM. MX2 is a dynamin-like GTPase that is an interferon-induced inhibitor of HIV-1 and other primate lentiviruses [70]. Impairing $M X 2$ 
function also leads to a delay in progression through the $\mathrm{G}_{1}-\mathrm{S}$ phase of the cell cycle [71]. Although Mangantig et al. found no association of MX2 rs45430 with Breslow thickness [47], other studies indicate $M X 2$ may influence melanoma progression [72,73]. MX2 rs45430 is in linkage disequilibrium with $M X 2 \mathrm{rs} 398206\left(\mathrm{D}^{\prime}=0.98\right.$ in the CEU population), and MX2 rs $45430^{*} \mathrm{~A}$ is strongly positively correlated with $M X 2 \mathrm{rs} 398206^{*} \mathrm{~A}$ [74]. Choi et al. identified MX2 rs398206 as a functional intronic variant that mediates Yingyang-1 (YY1) binding to increase $M X 2$ levels, with $M X 2$ rs398206*A driving significantly higher luciferase expression compared to the $C$ allele [72]. They further found that melanocyte-specific expression of human MX2 in a zebrafish model accelerated melanoma formation in a $B R A F V 600 \mathrm{E}$ background. Juraleviciute et al. found that primary melanomas homozygous for $M X 2$ rs45430*A had higher MX2 expression [73]. Interestingly, these authors found the effects of $M X 2$ expression on melanoma proliferation were context-dependent, with high expression in primary melanoma cell lines and melanocytes suppressing tumorigenesis, while downregulation in a subset of melanoma cell lines reduced proliferation. These differential effects in melanoma subsets may obscure associations in epidemiologic studies. Juraleviciute et al. also reported that $M X 2$ expression was significantly higher in tumors with TILs compared to tumors that had no TILs [73]. Here, we found no significant association with TILs for MX2 rs45430.

Our study's strengths are its international population-based design, large sample size, standardized pathology review, melanoma-specific survival, and comparatively long follow-up period ending before approvals of new systemic agents, checkpoint inhibitors, and targeted therapies that alter the natural course of the disease and improve overall survival [75]. Future studies examining melanoma-specific survival will likely be confounded by these new therapies. A limitation could be insufficient power to detect associations of SNPs with lower minor allele frequencies (e.g., SLC45A2 rs16891982, MAF 0.017). Another limitation is that our study only included participants with cutaneous melanoma, not mucosal [76,77] or uveal melanomas [78-80], which seemingly have different genetic landscapes.

\section{Conclusions}

Our findings indicate that few melanoma-risk variants are associated with tumor prognostic characteristics (Breslow thickness, presence of ulceration, presence of mitoses, or presence of TILs) or survival. However, further research investigating the associations of IRF4 rs12203592, CCND1 rs1485993, TERT rs2242652, and MX2 rs45430 with underlying biologic pathways related to tumor progression is warranted. Future studies of larger datasets that include subset analyses may help elucidate the relationship of melanoma-risk variants with tumor characteristics and survival.

Supplementary Materials: The following are available online at https://www.mdpi.com/article/ 10.3390/curroncol28060401/s1, Table S1: Genotype locations, minor/major alleles, minor allele frequencies, numbers of samples genotyped in the GEM study, and references for the association of the genotypes with melanoma; Table S2: Number of GEM participants successfully genotyped for each SNP and distribution of these participants by prognostic characteristics of primary melanoma tumor.

Author Contributions: Conceptualization: D.R.D. and N.E.T.; data curation: I.O., P.A.K., L.L., K.J.B., A.S., A.K., A.E.C., H.A.-C., S.B.G., R.P.G., R.Z., S.R., L.S., T.D., D.W.O., C.B.B., M.B. and N.E.T.; formal analysis: D.R.D. and N.E.T.; funding acquisition: I.O., A.E.C., H.A.-C., C.B.B., M.B. and N.E.T.; investigation: D.R.D. and N.E.T.; methodology: D.R.D., I.O. and N.E.T.; project administration: N.E.T.; resources: I.O.; software: L.L. and N.E.T.; supervision: N.E.T.; validation: I.O., P.A.K., C.B.B., M.B. and N.E.T.; visualization: D.R.D. and N.E.T.; writing—original draft: D.R.D. and N.E.T.; writing—review and editing: I.O., P.A.K., L.L., K.J.B., A.S., A.K., A.E.C., H.A.-C., S.B.G., R.P.G., R.Z., S.R., L.S., T.D., D.C.G., D.W.O., C.B.B., M.B. and N.E.T. All authors have read and agreed to the published version of the manuscript.

Funding: This work was supported by the National Cancer Institute (R01CA233524 to N.E. Thomas, M. Berwick, C.B. Begg, and H. Anton-Culver; P01CA206980 to N.E. Thomas and M. Berwick; R01CA112243 to N.E. Thomas; U01CA83180 and R01CA112524 to M. Berwick; R01CA098438 to C.B. 
Begg; R03CA125829 and R03CA173806 to I. Orlow; P30CA016086 to the University of North Carolina; and P30CA008748 to Memorial Sloan Kettering). A.E. Cust was supported by a NHMRC Career Development Fellowship.

Institutional Review Board Statement: The study was conducted according to the guidelines of the Declaration of Helsinki and approved by the Institutional Review Board (or Ethics Committee) of the University of New Mexico (protocol code CR00006961 and date of approval 24 January 2021).

Informed Consent Statement: The Genes, Environment, and Melanoma (GEM) Study obtained written informed consent from all participants involved in the study. This manuscript does not present identifying information for any participant and instead provides summary statistics.

Data Availability Statement: Data can be requested from Nancy E. Thomas or Marianne Berwick after review by the GEM Steering Committee.

Acknowledgments: GEM Study Group: Coordinating Center, Memorial Sloan Kettering Cancer Center, New York, NY (USA): Marianne Berwick, (PI, currently at the University of New Mexico, Albuquerque, NM), Colin Begg, (Co-PI), Irene Orlow, (Co-investigator), Klaus J. Busam, (Dermatopathologist), Isidora Autuori (Research Assistant), Audrey Mauguen, (Biostatistician). Germline DNA handling, and genotyping design and testing for this study specifically were completed by Pampa Roy (Senior Laboratory Technician), Sarah Yoo, (Senior Laboratory Technician), Ajay Sharma, (Senior Laboratory Technician), and Jaipreet Rayar, (Senior Laboratory Technician). University of New Mexico, Albuquerque, NM: Marianne Berwick, (PI), Li Luo, (Biostatistician), Tawny W. Boyce, (Data Manager). Study Centers: The University of Sydney and The Cancer Council New South Wales, Sydney, Australia: Anne E. Cust, (PI), Bruce K. Armstrong, (former PI), Anne Kricker, (former co-PI); Menzies Institute for Medical Research University of Tasmania, Hobart, Australia: Alison Venn, (current PI), Terence Dwyer, (PI, currently at University of Oxford, United Kingdom), Paul Tucker (Dermatopathologist); BC Cancer Research Centre, Vancouver, Canada: Richard P. Gallagher, (PI); Cancer Care Ontario, Toronto, Canada: Loraine D. Marrett, (PI), Lynn From, (Dermatopathologist); CPO, Center for Cancer Prevention, Torino, Italy: Roberto Zanetti, (PI), Stefano Rosso, (co-PI), Lidia Sacchetto, (Biostatistician); University of California, Irvine, CA: Hoda Anton-Culver, (PI); University of Michigan, Ann Arbor, MI: Stephen B. Gruber, (PI, currently at City of Hope National Medical Center, Duarte, CA), Shu-Chen Huang, (co-Investigator, joint at USC-University of Michigan); University of North Carolina, Chapel Hill, NC: Nancy E. Thomas, (PI), Kathleen Conway, (co-Investigator), David W. Ollila, (co-Investigator), Paul B. Googe, (Dermatopathologist), Sharon N. Edmiston, (Research Analyst), Honglin Hao (Laboratory Specialist), Eloise Parrish, (Laboratory Specialist), Sara E. Stevens, (Research Assistant), David C. Gibbs, (Research Assistant, currently at Emory University, Atlanta, GA); University of Pennsylvania, Philadelphia, PA: Timothy R. Rebbeck, (former PI), Peter A. Kanetsky, (PI, currently at H. Lee Moffitt Cancer Center \& Research Institute, Tampa, FL); UV data consultants: Julia Lee Taylor, and Sasha Madronich, National Centre for Atmospheric Research, Boulder, CO.

Conflicts of Interest: K. Busam has received minor royalties from editing a textbook with Elsevier. S.B. Gruber is the Co-Founder of Brogent International LLC. L. Sacchetto works as a biomarker statistician for Bayer AG. The remaining authors state no conflict of interest.

\section{References}

1. Law, M.H.; Macgregor, S.; Hayward, N.K. Melanoma genetics: Recent findings take us beyond well-traveled pathways. J. Investig. Dermatol. 2012, 132, 1763-1774. [CrossRef]

2. Gerstenblith, M.R.; Shi, J.; Landi, M.T. Genome-wide association studies of pigmentation and skin cancer: A review and meta-analysis. Pigment Cell Melanoma Res. 2010, 23, 587-606. [CrossRef]

3. Gibbs, D.C.; Orlow, I.; Kanetsky, P.A.; Luo, L.; Kricker, A.; Armstrong, B.K.; Anton-Culver, H.; Gruber, S.B.; Marrett, L.D.; Gallagher, R.P.; et al. Inherited genetic variants associated with occurrence of multiple primary melanoma. Cancer Epidemiol. Biomark. Prev. 2015, 24, 992-997. [CrossRef]

4. Amos, C.I.; Wang, L.E.; Lee, J.E.; Gershenwald, J.E.; Chen, W.V.; Fang, S.; Kosoy, R.; Zhang, M.; Qureshi, A.A.; Vattathil, S.; et al. Genome-wide association study identifies novel loci predisposing to cutaneous melanoma. Hum. Mol. Genet. 2011, 20, 5012-5023. [CrossRef] [PubMed]

5. Barrett, J.H.; Iles, M.M.; Harland, M.; Taylor, J.C.; Aitken, J.F.; Andresen, P.A.; Akslen, L.A.; Armstrong, B.K.; Avril, M.F.; Azizi, E.; et al. Genome-wide association study identifies three new melanoma susceptibility loci. Nat. Genet. 2011, 43, 1108-1113. [CrossRef] 
6. Chatzinasiou, F.; Lill, C.M.; Kypreou, K.; Stefanaki, I.; Nicolaou, V.; Spyrou, G.; Evangelou, E.; Roehr, J.T.; Kodela, E.; Katsambas, A.; et al. Comprehensive field synopsis and systematic meta-analyses of genetic association studies in cutaneous melanoma. $J$. Natl. Cancer Inst. 2011, 103, 1227-1235. [CrossRef] [PubMed]

7. Duffy, D.L.; Zhao, Z.Z.; Sturm, R.A.; Hayward, N.K.; Martin, N.G.; Montgomery, G.W. Multiple pigmentation gene polymorphisms account for a substantial proportion of risk of cutaneous malignant melanoma. J. Investig. Dermatol. 2010, 130, 520-528. [CrossRef] [PubMed]

8. Guedj, M.; Bourillon, A.; Combadières, C.; Rodero, M.; Dieudé, P.; Descamps, V.; Dupin, N.; Wolkenstein, P.; Aegerter, P.; Lebbe, C.; et al. Variants of the MATP/SLC45A2 gene are protective for melanoma in the French population. Hum. Mutat. 2008, 29, 1154-1160. [CrossRef] [PubMed]

9. Nan, H.; Kraft, P.; Hunter, D.J.; Han, J. Genetic variants in pigmentation genes, pigmentary phenotypes, and risk of skin cancer in Caucasians. Int. J. Cancer 2009, 125, 909-917. [CrossRef]

10. Sulem, P.; Gudbjartsson, D.F.; Stacey, S.N.; Helgason, A.; Rafnar, T.; Jakobsdottir, M.; Steinberg, S.; Gudjonsson, S.A.; Palsson, A.; Thorleifsson, G.; et al. Two newly identified genetic determinants of pigmentation in Europeans. Nat. Genet. 2008, 40, 835-837. [CrossRef]

11. Brown, K.M.; Macgregor, S.; Montgomery, G.W.; Craig, D.W.; Zhao, Z.Z.; Iyadurai, K.; Henders, A.K.; Homer, N.; Campbell, M.J.; Stark, M.; et al. Common sequence variants on 20q11.22 confer melanoma susceptibility. Nat. Genet. 2008, 40, 838-840. [CrossRef] [PubMed]

12. Bishop, D.T.; Demenais, F.; Iles, M.M.; Harland, M.; Taylor, J.C.; Corda, E.; Randerson-Moor, J.; Aitken, J.F.; Avril, M.F.; Azizi, E.; et al. Genome-wide association study identifies three loci associated with melanoma risk. Nat. Genet. 2009, 41, 920-925. [CrossRef] [PubMed]

13. Fang, S.; Han, J.; Zhang, M.; Wang, L.E.; Wei, Q.; Amos, C.I.; Lee, J.E. Joint effect of multiple common SNPs predicts melanoma susceptibility. PLoS ONE 2013, 8, e85642. [CrossRef] [PubMed]

14. Orlow, I.; Satagopan, J.M.; Berwick, M.; Enriquez, H.L.; White, K.A.; Cheung, K.; Dusza, S.W.; Oliveria, S.A.; Marchetti, M.A.; Scope, A.; et al. Genetic factors associated with naevus count and dermoscopic patterns: Preliminary results from the Study of Nevi in Children (SONIC). Br. J. Dermatol. 2015, 172, 1081-1089. [CrossRef]

15. Nan, H.; Xu, M.; Zhang, J.; Zhang, M.; Kraft, P.; Qureshi, A.A.; Chen, C.; Guo, Q.; Hu, F.B.; Rimm, E.B.; et al. Genome-wide association study identifies nidogen 1 (NID1) as a susceptibility locus to cutaneous nevi and melanoma risk. Hum. Mol. Genet. 2011, 20, 2673-2679. [CrossRef]

16. Falchi, M.; Bataille, V.; Hayward, N.K.; Duffy, D.L.; Bishop, J.A.; Pastinen, T.; Cervino, A.; Zhao, Z.Z.; Deloukas, P.; Soranzo, N.; et al. Genome-wide association study identifies variants at 9p21 and 22q13 associated with development of cutaneous nevi. Nat. Genet. 2009, 41, 915-919. [CrossRef] [PubMed]

17. Newton-Bishop, J.A.; Chang, Y.M.; Iles, M.M.; Taylor, J.C.; Bakker, B.; Chan, M.; Leake, S.; Karpavicius, B.; Haynes, S.; Fitzgibbon, E.; et al. Melanocytic nevi, nevus genes, and melanoma risk in a large case-control study in the United Kingdom. Cancer Epidemiol. Biomark. Prev. 2010, 19, 2043-2054. [CrossRef] [PubMed]

18. Maccioni, L.; Rachakonda, P.S.; Bermejo, J.L.; Planelles, D.; Requena, C.; Hemminki, K.; Nagore, E.; Kumar, R. Variants at the 9p21 locus and melanoma risk. BMC Cancer 2013, 13, 325. [CrossRef]

19. Liu, F.; Visser, M.; Duffy, D.L.; Hysi, P.G.; Jacobs, L.C.; Lao, O.; Zhong, K.; Walsh, S.; Chaitanya, L.; Wollstein, A.; et al. Genetics of skin color variation in Europeans: Genome-wide association studies with functional follow-up. Hum. Genet. 2015, 134, 823-835. [CrossRef] [PubMed]

20. Peña-Chilet, M.; Blanquer-Maceiras, M.; Ibarrola-Villava, M.; Martinez-Cadenas, C.; Martin-Gonzalez, M.; Gomez-Fernandez, C.; Mayor, M.; Aviles, J.A.; Lluch, A.; Ribas, G. Genetic variants in PARP1 (rs3219090) and IRF4 (rs12203592) genes associated with melanoma susceptibility in a Spanish population. BMC Cancer 2013,13, 160. [CrossRef]

21. Duffy, D.L.; Iles, M.M.; Glass, D.; Zhu, G.; Barrett, J.H.; Höiom, V.; Zhao, Z.Z.; Sturm, R.A.; Soranzo, N.; Hammond, C.; et al. IRF4 variants have age-specific effects on nevus count and predispose to melanoma. Am. J. Hum. Genet. 2010, 87, 6-16. [CrossRef] [PubMed]

22. Han, J.; Qureshi, A.A.; Nan, H.; Zhang, J.; Song, Y.; Guo, Q.; Hunter, D.J. A germline variant in the interferon regulatory factor 4 gene as a novel skin cancer risk locus. Cancer Res. 2011, 71, 1533-1539. [CrossRef] [PubMed]

23. Zhang, M.; Song, F.; Liang, L.; Nan, H.; Zhang, J.; Liu, H.; Wang, L.E.; Wei, Q.; Lee, J.E.; Amos, C.I.; et al. Genome-wide association studies identify several new loci associated with pigmentation traits and skin cancer risk in European Americans. Hum. Mol. Genet. 2013, 22, 2948-2959. [CrossRef] [PubMed]

24. Nan, H.; Qureshi, A.A.; Prescott, J.; De Vivo, I.; Han, J. Genetic variants in telomere-maintaining genes and skin cancer risk. Hum. Genet. 2011, 129, 247-253. [CrossRef]

25. Gershenwald, J.E.; Scolyer, R.A.; Hess, K.R.; Thompson, J.F.; Long, G.V.; Ross, M.I.; Lazar, A.J.; Atkins, M.B.; Balch, C.M.; Barnhill, R.L.; et al. Melanoma of the skin. In AJCC Cancer Staging Manual, 8th ed.; Amin, M.B., Edge, S.B., Greene, F.L., Byrd, D.R., Brookland, R.K., Washington, M.K., Gershenwald, J.E., Compton, C.C., Hess, K.R., Sullivan, D.C., et al., Eds.; Springer International Publishing: New York, NY, USA, 2017; pp. 563-585.

26. Kashani-Sabet, M.; Miller, J.R., 3rd; Lo, S.; Nosrati, M.; Stretch, J.R.; Shannon, K.F.; Spillane, A.J.; Saw, R.P.M.; Cleaver, J.E.; Kim, K.B.; et al. Reappraisal of the prognostic significance of mitotic rate supports its reincorporation into the melanoma staging system. Cancer 2020, 126, 4717-4725. [CrossRef] 
27. Namikawa, K.; Aung, P.P.; Gershenwald, J.E.; Milton, D.R.; Prieto, V.G. Clinical impact of ulceration width, lymphovascular invasion, microscopic satellitosis, perineural invasion, and mitotic rate in patients undergoing sentinel lymph node biopsy for cutaneous melanoma: A retrospective observational study at a comprehensive cancer center. Cancer Med. 2018, 7, 583-593. [CrossRef] [PubMed]

28. Clemente, C.G.; Mihm, M.C., Jr.; Bufalino, R.; Zurrida, S.; Collini, P.; Cascinelli, N. Prognostic value of tumor infiltrating lymphocytes in the vertical growth phase of primary cutaneous melanoma. Cancer 1996, 77, 1303-1310. [CrossRef]

29. Azimi, F.; Scolyer, R.A.; Rumcheva, P.; Moncrieff, M.; Murali, R.; McCarthy, S.W.; Saw, R.P.; Thompson, J.F. Tumor-infiltrating lymphocyte grade is an independent predictor of sentinel lymph node status and survival in patients with cutaneous melanoma. J. Clin. Oncol. 2012, 30, 2678-2683. [CrossRef] [PubMed]

30. Thomas, N.E.; Busam, K.J.; From, L.; Kricker, A.; Armstrong, B.K.; Anton-Culver, H.; Gruber, S.B.; Gallagher, R.P.; Zanetti, R.; Rosso, S.; et al. Tumor-infiltrating lymphocyte grade in primary melanomas is independently associated with melanoma-specific survival in the population-based genes, environment and melanoma study. J. Clin. Oncol. 2013, 31, 4252-4259. [CrossRef]

31. Begg, C.B.; Hummer, A.J.; Mujumdar, U.; Armstrong, B.K.; Kricker, A.; Marrett, L.D.; Millikan, R.C.; Gruber, S.B.; Culver, H.A.; Zanetti, R.; et al. A design for cancer case-control studies using only incident cases: Experience with the GEM study of melanoma. Int. J. Epidemiol. 2006, 35, 756-764. [CrossRef]

32. Thomas, N.E.; Kricker, A.; From, L.; Busam, K.; Millikan, R.C.; Ritchey, M.E.; Armstrong, B.K.; Lee-Taylor, J.; Marrett, L.D.; Anton-Culver, H.; et al. Associations of cumulative sun exposure and phenotypic characteristics with histologic solar elastosis. Cancer Epidemiol. Biomark. Prev. 2010, 19, 2932-2941. [CrossRef]

33. Thomas, N.E.; Kricker, A.; Waxweiler, W.T.; Dillon, P.M.; Busman, K.J.; From, L.; Groben, P.A.; Armstrong, B.K.; Anton-Culver, H.; Gruber, S.B.; et al. Comparison of clinicopathologic features and survival of histopathologically amelanotic and pigmented melanomas: A population-based study. JAMA Dermatol. 2014, 150, 1306-1314. [CrossRef]

34. Thomas, N.E.; Edmiston, S.N.; Alexander, A.; Groben, P.A.; Parrish, E.; Kricker, A.; Armstrong, B.K.; Anton-Culver, H.; Gruber, S.B.; From, L.; et al. Association Between NRAS and BRAF Mutational Status and Melanoma-Specific Survival Among Patients With Higher-Risk Primary Melanoma. JAMA Oncol. 2015, 1, 359-368. [CrossRef] [PubMed]

35. Clark, W.H., Jr.; From, L.; Bernardino, E.A.; Mihm, M.C. The histogenesis and biologic behavior of primary human malignant melanomas of the skin. Cancer Res. 1969, 29, 705-727. [PubMed]

36. McGovern, V.J.; Mihm, M.C., Jr.; Bailly, C.; Booth, J.C.; Clark, W.H., Jr.; Cochran, A.J.; Hardy, E.G.; Hicks, J.D.; Levene, A.; Lewis, M.G.; et al. The classification of malignant melanoma and its histologic reporting. Cancer 1973, 32, 1446-1457. [CrossRef]

37. Piris, A.; Mihm, M.C., Jr.; Duncan, L.M. AJCC melanoma staging update: Impact on dermatopathology practice and patient management. J. Cutan. Pathol. 2011, 38, 394-400. [CrossRef] [PubMed]

38. Clark, W.H., Jr.; Elder, D.E.; Guerry, D.T.; Braitman, L.E.; Trock, B.J.; Schultz, D.; Synnestvedt, M.; Halpern, A.C. Model predicting survival in stage I melanoma based on tumor progression. J. Natl. Cancer Inst. 1989, 81, 1893-1904. [CrossRef]

39. Elder, D.E.; Guerry, D.t.; VanHorn, M.; Hurwitz, S.; Zehngebot, L.; Goldman, L.I.; LaRossa, D.; Hamilton, R.; Bondi, E.E.; Clark, W.H., Jr. The role of lymph node dissection for clinical stage I malignant melanoma of intermediate thickness (1.51-3.99 mm). Cancer 1985, 56, 413-418. [CrossRef]

40. Elder, D.E.; Gimotty, P.A.; Guerry, D. Cutaneous melanoma: Estimating survival and recurrence risk based on histopathologic features. Dermatol. Ther. 2005, 18, 369-385. [CrossRef]

41. Orlow, I.; Roy, P.; Reiner, A.S.; Yoo, S.; Patel, H.; Paine, S.; Armstrong, B.K.; Kricker, A.; Marrett, L.D.; Millikan, R.C.; et al. Vitamin D receptor polymorphisms in patients with cutaneous melanoma. Int. J. Cancer 2012, 130, 405-418. [CrossRef]

42. Gibbs, D.C.; Orlow, I.; Bramson, J.I.; Kanetsky, P.A.; Luo, L.; Kricker, A.; Armstrong, B.K.; Anton-Culver, H.; Gruber, S.B.; Marrett, L.D.; et al. Association of Interferon Regulatory Factor-4 Polymorphism rs12203592 With Divergent Melanoma Pathways. J. Natl. Cancer Inst. 2016, 108, djw004. [CrossRef]

43. He, Q.; Avery, C.L.; Lin, D.Y. A general framework for association tests with multivariate traits in large-scale genomics studies. Genet. Epidemiol. 2013, 37, 759-767. [CrossRef]

44. Lin, D.Y. An efficient Monte Carlo approach to assessing statistical significance in genomic studies. Bioinformatics 2005, 21, 781-787. [CrossRef]

45. Gibbs, D.C.; Ward, S.V.; Orlow, I.; Cadby, G.; Kanetsky, P.A.; Luo, L.; Busam, K.J.; Kricker, A.; Armstrong, B.K.; Cust, A.E.; et al. Functional melanoma-risk variant IRF4 rs12203592 associated with Breslow thickness: A pooled international study of primary melanomas. Br. J. Dermatol. 2017, 177, e180-e182. [CrossRef]

46. Ward, S.V.; Gibbs, D.C.; Orlow, I.; Thomas, N.E.; Kanetsky, P.A.; Luo, L.; Cust, A.E.; Anton-Culver, H.; Gruber, S.B.; Gallagher, R.P.; et al. Association of IRF4 single-nucleotide polymorphism rs12203592 with melanoma-specific survival. Br. J. Dermatol. 2020, 183, 163-165. [CrossRef]

47. Mangantig, E.; MacGregor, S.; Iles, M.M.; Scolyer, R.A.; Cust, A.E.; Hayward, N.K.; Montgomery, G.W.; Duffy, D.L.; Thompson, J.F.; Henders, A.; et al. Germline variants are associated with increased primary melanoma tumor thickness at diagnosis. Hum. Mol. Genet. 2021, 29, 3578-3587. [CrossRef]

48. Potrony, M.; Rebollo-Morell, A.; Giménez-Xavier, P.; Zimmer, L.; Puig-Butille, J.A.; Tell-Marti, G.; Sucker, A.; Badenas, C.; Carrera, C.; Malvehy, J.; et al. IRF4 rs12203592 functional variant and melanoma survival. Int. J. Cancer 2017, 140, 1845-1849. [CrossRef] [PubMed]

49. Paun, A.; Pitha, P.M. The IRF family, revisited. Biochimie 2007, 89, 744-753. [CrossRef] [PubMed] 
50. Do, T.N.; Ucisik-Akkaya, E.; Davis, C.F.; Morrison, B.A.; Dorak, M.T. An intronic polymorphism of IRF4 gene influences gene transcription in vitro and shows a risk association with childhood acute lymphoblastic leukemia in males. Biochim. Biophys. Acta 2010, 1802, 292-300. [CrossRef] [PubMed]

51. Hrdlicková, R.; Nehyba, J.; Bose, H.R., Jr. Regulation of telomerase activity by interferon regulatory factors 4 and 8 in immune cells. Mol. Cell Biol. 2009, 29, 929-941. [CrossRef]

52. Smith, L.L.; Coller, H.A.; Roberts, J.M. Telomerase modulates expression of growth-controlling genes and enhances cell proliferation. Nat. Cell Biol. 2003, 5, 474-479. [CrossRef] [PubMed]

53. Zheng, Y.; Chaudhry, A.; Kas, A.; deRoos, P.; Kim, J.M.; Chu, T.T.; Corcoran, L.; Treuting, P.; Klein, U.; Rudensky, A.Y. Regulatory T-cell suppressor program co-opts transcription factor IRF4 to control T(H)2 responses. Nature 2009, 458, 351-356. [CrossRef] [PubMed]

54. Nam, S.; Kang, K.; Cha, J.S.; Kim, J.W.; Lee, H.G.; Kim, Y.; Yang, Y.; Lee, M.S.; Lim, J.S. Interferon regulatory factor 4 (IRF4) controls myeloid-derived suppressor cell (MDSC) differentiation and function. J. Leukoc. Biol. 2016, 100, 1273-1284. [CrossRef]

55. Metzger, P.; Kirchleitner, S.V.; Boehmer, D.F.R.; Hörth, C.; Eisele, A.; Ormanns, S.; Gunzer, M.; Lech, M.; Lauber, K.; Endres, S.; et al. Systemic but not MDSC-specific IRF4 deficiency promotes an immunosuppressed tumor microenvironment in a murine pancreatic cancer model. Cancer Immunol. Immunother. 2020, 69, 2101-2112. [CrossRef]

56. Kim, J.K.; Diehl, J.A. Nuclear cyclin D1: An oncogenic driver in human cancer. J. Cell. Physiol. 2009, 220, 292-296. [CrossRef]

57. Fu, M.; Wang, C.; Li, Z.; Sakamaki, T.; Pestell, R.G. Minireview: Cyclin D1: Normal and abnormal functions. Endocrinology 2004, 145, 5439-5447. [CrossRef]

58. González-Ruiz, L.; González-Moles, M.; González-Ruiz, I.; Ruiz-Ávila, I.; Ayén, Á.; Ramos-García, P. An update on the implications of cyclin D1 in melanomas. Pigment Cell Melanoma Res. 2020, 33, 788-805. [CrossRef]

59. Law, M.H.; Montgomery, G.W.; Brown, K.M.; Martin, N.G.; Mann, G.J.; Hayward, N.K.; MacGregor, S. Meta-analysis combining new and existing data sets confirms that the TERT-CLPTM1L locus influences melanoma risk. J. Investig. Dermatol. 2012, 132, 485-487. [CrossRef]

60. Rafnar, T.; Sulem, P.; Stacey, S.N.; Geller, F.; Gudmundsson, J.; Sigurdsson, A.; Jakobsdottir, M.; Helgadottir, H.; Thorlacius, S.; Aben, K.K.; et al. Sequence variants at the TERT-CLPTM1L locus associate with many cancer types. Nat. Genet. 2009, 41, 221-227. [CrossRef]

61. Chiba, K.; Lorbeer, F.K.; Shain, A.H.; McSwiggen, D.T.; Schruf, E.; Oh, A.; Ryu, J.; Darzacq, X.; Bastian, B.C.; Hockemeyer, D. Mutations in the promoter of the telomerase gene TERT contribute to tumorigenesis by a two-step mechanism. Science 2017, 357, 1416-1420. [CrossRef] [PubMed]

62. Heidenreich, B.; Nagore, E.; Rachakonda, P.S.; Garcia-Casado, Z.; Requena, C.; Traves, V.; Becker, J.; Soufir, N.; Hemminki, K.; Kumar, R. Telomerase reverse transcriptase promoter mutations in primary cutaneous melanoma. Nat. Commun. $2014,5,3401$. [CrossRef]

63. Griewank, K.G.; Murali, R.; Puig-Butille, J.A.; Schilling, B.; Livingstone, E.; Potrony, M.; Carrera, C.; Schimming, T.; Möller, I.; Schwamborn, M.; et al. TERT promoter mutation status as an independent prognostic factor in cutaneous melanoma. J. Natl. Cancer Inst. 2014, 106, dju246. [CrossRef] [PubMed]

64. Pópulo, H.; Boaventura, P.; Vinagre, J.; Batista, R.; Mendes, A.; Caldas, R.; Pardal, J.; Azevedo, F.; Honavar, M.; Guimarães, I.; et al. TERT promoter mutations in skin cancer: The effects of sun exposure and X-irradiation. J. Investig. Dermatol. 2014, 134, $2251-2257$. [CrossRef] [PubMed]

65. Egberts, F.; Bohne, A.S.; Krüger, S.; Hedderich, J.; Rompel, R.; Haag, J.; Röcken, C.; Hauschild, A. Varying Mutational Alterations in Multiple Primary Melanomas. J. Mol. Diagn. 2016, 18, 75-83. [CrossRef]

66. Nagore, E.; Heidenreich, B.; Rachakonda, S.; Garcia-Casado, Z.; Requena, C.; Soriano, V.; Frank, C.; Traves, V.; Quecedo, E.; Sanjuan-Gimenez, J.; et al. TERT promoter mutations in melanoma survival. Int. J. Cancer 2016, 139, 75-84. [CrossRef]

67. Ekedahl, H.; Lauss, M.; Olsson, H.; Griewank, K.G.; Schadendorf, D.; Ingvar, C.; Jönsson, G. High TERT promoter mutation frequency in non-acral cutaneous metastatic melanoma. Pigment Cell Melanoma Res. 2016, 29, 598-600. [CrossRef] [PubMed]

68. Lade-Keller, J.; Yuusufi, S.; Riber-Hansen, R.; Steiniche, T.; Stougaard, M. Telomerase reverse transcriptase promoter mutations and solar elastosis in cutaneous melanoma. Melanoma Res. 2018, 28, 398-409. [CrossRef]

69. Thomas, N.E.; Edmiston, S.N.; Tsai, Y.S.; Parker, J.S.; Googe, P.B.; Busam, K.J.; Scott, G.A.; Zedek, D.C.; Parrish, E.A.; Hao, H.; et al. Utility of TERT Promoter Mutations for Cutaneous Primary Melanoma Diagnosis. Am. J. Dermatopathol. 2019, 41, $264-272$. [CrossRef]

70. Haller, O.; Staeheli, P.; Schwemmle, M.; Kochs, G. Mx GTPases: Dynamin-like antiviral machines of innate immunity. Trends Microbiol. 2015, 23, 154-163. [CrossRef]

71. King, M.C.; Raposo, G.; Lemmon, M.A. Inhibition of nuclear import and cell-cycle progression by mutated forms of the dynamin-like GTPase MxB. Proc. Natl. Acad. Sci. USA 2004, 101, 8957-8962. [CrossRef]

72. Choi, J.; Zhang, T.; Vu, A.; Ablain, J.; Makowski, M.M.; Colli, L.M.; Xu, M.; Hennessey, R.C.; Yin, J.; Rothschild, H.; et al. Massively parallel reporter assays of melanoma risk variants identify MX2 as a gene promoting melanoma. Nat. Commun. 2020, 11, 2718. [CrossRef]

73. Juraleviciute, M.; Pozniak, J.; Nsengimana, J.; Harland, M.; Randerson-Moor, J.; Wernhoff, P.; Bassarova, A.; Øy, G.F.; Trøen, G.; Flørenes, V.A.; et al. MX 2 is a novel regulator of cell cycle in melanoma cells. Pigment Cell Melanoma Res. 2020, 33, 446-457. [CrossRef] [PubMed] 
74. National Institutes of Health; National Cancer Institute. Division of Cancer Epidemiology \& Genetics. LDpair Tool. 2021. Available online: https:/ /ldlink.nci.nih.gov/?tab=ldpair (accessed on 4 June 2021).

75. Franklin, C.; Livingstone, E.; Roesch, A.; Schilling, B.; Schadendorf, D. Immunotherapy in melanoma: Recent advances and future directions. Eur. J. Surg. Oncol. 2017, 43, 604-611. [CrossRef] [PubMed]

76. Newell, F.; Kong, Y.; Wilmott, J.S.; Johansson, P.A.; Ferguson, P.M.; Cui, C.; Li, Z.; Kazakoff, S.H.; Burke, H.; Dodds, T.J.; et al. Whole-genome landscape of mucosal melanoma reveals diverse drivers and therapeutic targets. Nat. Commun. 2019, 10, 3163. [CrossRef]

77. Broit, N.; Johansson, P.A.; Rodgers, C.B.; Walpole, S.T.; Newell, F.; Hayward, N.K.; Pritchard, A.L. Meta-Analysis and Systematic Review of the Genomics of Mucosal Melanoma. Mol. Cancer Res. 2021, 19, 991-1004. [CrossRef] [PubMed]

78. Basile, M.S.; Mazzon, E.; Russo, A.; Mammana, S.; Longo, A.; Bonfiglio, V.; Fallico, M.; Caltabiano, R.; Fagone, P.; Nicoletti, F.; et al. Differential modulation and prognostic values of immune-escape genes in uveal melanoma. PLoS ONE 2019, 14, e0210276. [CrossRef]

79. Barbagallo, C.; Caltabiano, R.; Broggi, G.; Russo, A.; Puzzo, L.; Avitabile, T.; Longo, A.; Reibaldi, M.; Barbagallo, D.; Di Pietro, C.; et al. LncRNA LINC00518 Acts as an Oncogene in Uveal Melanoma by Regulating an RNA-Based Network. Cancers 2020, 12, 3867. [CrossRef]

80. Mastronikolis, S.; Adamopoulou, M.; Papouliakos, S.; Manoli, A.; Katsinis, S.; Makri, O.; Monastirioti, A.E.; Tsiambas, E.; Georgakopoulos, C. Mutational landscape in Uveal Melanoma. J. BU ON 2021, 26, 1194-1197. 